\title{
Transgenic up-regulation of alpha-CaMKII in forebrain leads to increased anxiety-like behaviors and aggression
} Shunsuke Hasegawa1, Takahiro Furuichi' ${ }^{1}$, Taro Yoshida1, Kengo Endoh ${ }^{1}$, Kenichi Kato ${ }^{1}$, Megumi Sado1, Ryouta Maeda1 , Aya Kitamoto1, Takahisa Miyao ${ }^{1}$, Ryosuke Suzuki ${ }^{1}$, Seiichi Homma ${ }^{1}$, Shoichi Masushige ${ }^{1}$, Yasushi Kajii $^{2}$ and Satoshi Kida*1,3

\author{
Address: ${ }^{1}$ Department of Bioscience, Faculty of Applied Bioscience, Tokyo University of Agriculture, Tokyo 156-8502, Japan, ${ }^{2}$ Pharmacology \\ Laboratory Mitsubishi Tanabe Pharma Corporation, Yokohama 227-0033, Japan and ${ }^{3}$ Core Research for Evolutional Science and Technology, \\ Japan Science and Technology Agency, Saitama 332-0012, Japan \\ Email: Shunsuke Hasegawa - 56060003@nodai.ac.jp; Takahiro Furuichi - furu1232000@yahoo.co.jp; Taro Yoshida - 56060006@nodai.ac.jp; \\ Kengo Endoh - 55080013@nodai.ac.jp; Kenichi Kato - shutenndouji@excite.co.jp; Megumi Sado - sp3h9t59@triton.ocn.ne.jp; \\ Ryouta Maeda - Maeda_Ryouta@takeda.co.jp; Aya Kitamoto - loipero0503@yahoo.co.jp; Takahisa Miyao - 55070025@nodai.ac.jp; \\ Ryosuke Suzuki-55070032@nodai.ac.jp; Seiichi Homma - s3homma@nodai.ac.jp; Shoichi Masushige - s-mass@tsc-05.ac.jp; \\ Yasushi Kajii - Kajii.Yasushi@mb.mt-pharma.co.jp; Satoshi Kida* - kida@nodai.ac.jp \\ * Corresponding author
}

Published: 4 March 2009

Molecular Brain 2009, 2:6 doi:10.1186/1756-6606-2-6

This article is available from: http://www.molecularbrain.com/content/2/l/6

(c) 2009 Hasegawa et al; licensee BioMed Central Ltd.

This is an Open Access article distributed under the terms of the Creative Commons Attribution License (http://creativecommons.org/licenses/by/2.0), which permits unrestricted use, distribution, and reproduction in any medium, provided the original work is properly cited.

\begin{abstract}
Background: Previous studies have demonstrated essential roles for alpha-calcium/calmodulindependent protein kinase II (alpha-CaMKII) in learning, memory and long-term potentiation (LTP). However, previous studies have also shown that alpha-CaMKII (+/-) heterozygous knockout mice display a dramatic decrease in anxiety-like and fearful behaviors, and an increase in defensive aggression. These findings indicated that alpha-CaMKII is important not only for learning and memory but also for emotional behaviors. In this study, to understand the roles of alpha-CaMKII in emotional behavior, we generated transgenic mice overexpressing alpha-CaMKII in the forebrain and analyzed their behavioral phenotypes.

Results: We generated transgenic mice overexpressing alpha-CaMKII in the forebrain under the control of the alpha-CaMKII promoter. In contrast to alpha-CaMKII (+/-) heterozygous knockout mice, alphaCaMKII overexpressing mice display an increase in anxiety-like behaviors in open field, elevated zero maze, light-dark transition and social interaction tests, and a decrease in locomotor activity in their home cages and novel environments; these phenotypes were the opposite to those observed in alpha-CaMKII $(+/-)$ heterozygous knockout mice. In addition, similarly with alpha-CaMKII (+/-) heterozygous knockout mice, alpha-CaMKII overexpressing mice display an increase in aggression. However, in contrast to the increase in defensive aggression observed in alpha-CaMKII (+/-) heterozygous knockout mice, alpha-CaMKII overexpressing mice display an increase in offensive aggression.

Conclusion: Up-regulation of alpha-CaMKII expression in the forebrain leads to an increase in anxietylike behaviors and offensive aggression. From the comparisons with previous findings, we suggest that the expression levels of alpha-CaMKII are associated with the state of emotion; the expression level of alphaCaMKII positively correlates with the anxiety state and strongly affects aggressive behavior.
\end{abstract}




\section{Background}

Alpha-CaMKII is a Ser/Thr protein kinase that is abundantly expressed in the forebrain [1-5]. In response to an increase in the intracellular concentration of $\mathrm{Ca}^{2+}$, alphaCaMKII is activated through the binding of $\mathrm{Ca}^{2+} /$ calmodulin (CaM), and then phosphorylates target proteins to activate or inactivate these proteins [1-5]. Furthermore, the prolonged activation of alpha-CaMKII by $\mathrm{Ca}^{2+} / \mathrm{CaM}$ results in the intramolecular autophosphorylation of Thr residues such as T286, T305, and T306 [1-5]. Autophosphorylation of T286 leads to a decrease in the dissociation of bound $\mathrm{Ca}^{2+} / \mathrm{CaM}$ and continuous partial activation even after the dissociation of $\mathrm{Ca}^{2+} / \mathrm{CaM}$, thereby prolonging its activation. Thus, a transient increase in intracellular $\mathrm{Ca}^{2+}$ can result in prolonged alpha-CaMKII activation until T286 is dephosphorylated by protein phosphatases [1-5].

Genetic studies have examined the effects of the loss or gain of function of alpha-CaMKII [2-15]. Genetic deletion of alpha-CaMKII impaired hippocampus-dependent spatial learning in homozygous knockout mice [8]. Furthermore, mice with a Thr286 to Ala (T286A) point mutation leading to a lack of the autonomous activity of this kinase have also shown severe impairments of spatial learning [9]. These findings indicate that alpha-CaMKII is an essential molecule involved in learning and memory. However, several studies have shown that overexpression of alphaCaMKII or an increase in alpha-CaMKII activity does not simply lead to an enhanced ability in learning/memory [11-15]. Transgenic mice overexpressing wild-type or a constitutively active mutant (T286D) of alpha-CaMKII in the forebrain showed deficits in learning/memory [11$15]$.

Interestingly, a previous study also showed that mutant mice with a deletion of the alpha-CaMKII gene display abnormal emotional behavior [16]. Both alpha-CaMKII (/-) homozygous and (+/-) heterozygous knockout mice exhibited attenuated fear behavior. Furthermore, heterozygous knockout mice showed increased defensive aggression but normal offensive aggression [16], which is consistent with a reduction in fear behavior. A recent study extended these observations; heterozygous knockout mice displayed a dramatic decrease in anxiety-like behaviors and symptoms of psychiatric disorders such as bipolar disorders and schizophrenia [17]. These findings indicate that alpha-CaMKII plays critical roles in not only learning and memory, but also in emotional behaviors including anxiety, the fear response and aggression $[16,17]$.

In this study, to understand the role of alpha-CaMKII in emotional behavior, we generated transgenic mice expressing wild-type alpha-CaMKII in the forebrain under the control of the alpha-CaMKII promoter. These trans- genic mice displayed an increase in anxiety-like behavior and a decrease in locomotor activity, which are the opposite phenotypes to those observed in alpha-CaMKII (+/-) heterozygous knockout mice [16,17]. Interestingly, in contrast to the observations in alpha-CaMKII (+/-) heterozygous knockout mice, alpha-CaMKII overexpressing mice exhibited increased offensive but normal defensive aggression. Taken together with previous observations, our findings suggest that the expression level of alphaCaMKII plays important roles in emotional behavior, and especially, that the expression level of alpha-CaMKII positively correlates with anxiety-like behaviors.

\section{Results}

\section{Generation of alpha-CaMKII overexpressing mice}

To investigate the role of alpha-CaMKII in emotional behavior, we generated transgenic mice overexpressing wild-type alpha-CaMKII in the forebrain using the alphaCaMKII promoter $[12-14,18,19]$. We first constructed a transgene that contained the alpha-CaMKII promoter, a hybrid intron in the 5'-untranslated leader sequence, the coding region of alpha-CaMKII fused with the FLAG-tag sequence at the $\mathrm{N}$-terminus, and a polyadenylation signal (Figure 1A). The alpha-CaMKII promoter used in this study has been known to exhibit strong activity in regions of the forebrain including the hippocampus, cortex and striatum [12-14,18,19].

We next performed expression analyses in the transgenic alpha-CaMKII mice. In situ hybridization analyses revealed high levels of expression of transgene mRNA in regions of the forebrain including the CA1, CA3, and dentate gyrus areas of the hippocampus, basolateral and lateral areas of amygdala and cortical and prefrontal regions (Figure 1B). To compare the expression level of alphaCaMKII between transgenic and wild-type (WT) littermates, we analyzed the levels of alpha-CaMKII in the forebrain using Western blotting. Quantitative immunoblotting with antibodies recognizing alpha-CaMKII revealed 2-2.5 fold higher expression of alpha-CaMKII in the hippocampus and cortex of transgenic mice compared with WT littermates $\left(\mathrm{HP}, \mathrm{F}_{1,12}=5.513, \mathrm{P}=\right.$ $0.0368 ; \mathrm{CO}, \mathrm{F}_{1,12}=6.382, \mathrm{P}=0.0266$; Figure $\left.1 \mathrm{C}\right)$. Interestingly, no difference was observed in the level of the phosphorylated form of alpha-CaMKII at T286 between the alpha-CaMKII overexpressing mice and WT littermates (Figure 1C). This is a similar observation with previous findings in which alpha-CaMKII (+/-) heterozygous knockout mice display comparable levels of phosphorylated alpha-CaMKII at T286 with WT littermates [17]. Some compensatory mechanisms might exist by which the activity of alpha-CaMKII is kept at the constant basal level.

Previous study has shown that alpha-CaMKII (+/-) heterozygous knockout mice displayed abnormalities of gene 
A

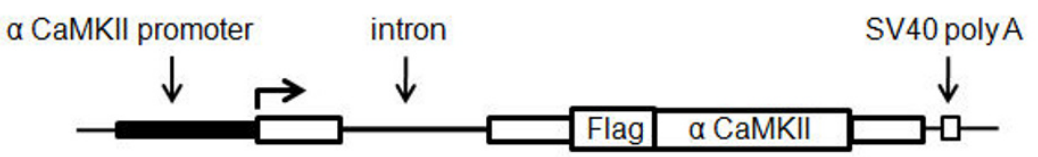

B

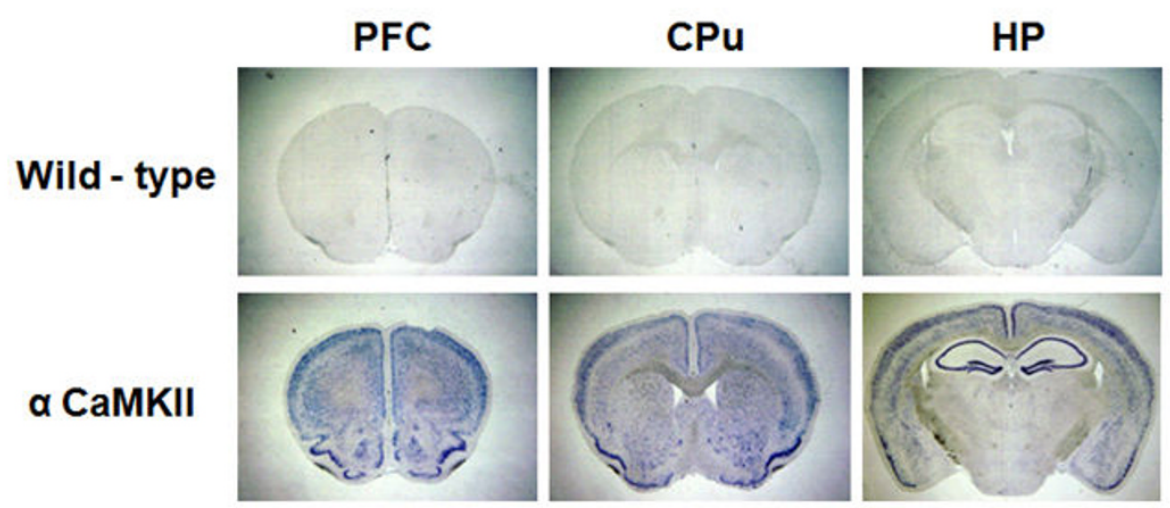

C

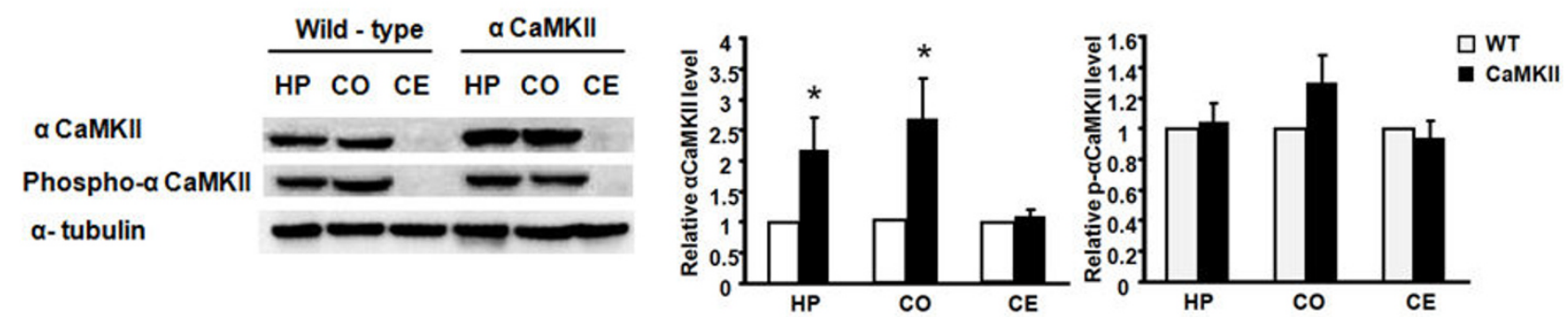

\section{Figure I}

Generation and expression analyses of alpha-CaMKII overexpressing mice. (A) Schematic representation of the alpha-CaMKII transgenic construct. (B) In situ hybridization analyses of transgene mRNA expression in the alpha-CaMKII overexpressing and WT mice. (C) Western blot analyses of the levels of alpha-CaMKII and phosphorylated alpha-CaMKII at T286 in the hippocampus, cortex, and cerebellum of alpha-CaMKII overexpressing and WT mice. Levels of alpha-CaMKII and phosphorylated alpha-CaMKII were normalized according to the levels of alpha-tubulin (hippocampus, WT, $n=7 ;$ alpha-CaMKII, $n=7$; cortex, WT, $n=7$; alpha-CaMKII, $n=7$; cerebellum, WT, $n=5$; alpha-CaMKII, $n=5$ ). The asterisk indicates statistical significance at $P<0.05$.

expression profiles in hippocampus [17]. To examine effects of overexpression of alpha-CaMKII at the molecular level, we analyzed hippocampal expression levels of five genes that decreased in hippocampus of alpha-CaMKII (+/-) heterozygous knockout mice (Additional file 1, Figure S1). Analyses using quantitative PCR showed that alpha-CaMKII overexpressing mice exhibited a significant decrease in nephronectin (Npnt) mRNA level $\left(\mathrm{F}_{1,8}=\right.$ 6.409, $\mathrm{P}=0.0352$; Additional file 1 , Figure $\mathrm{S} 1 \mathrm{~A}$ ) and trends towards to a decrease in mRNA levels of tryptophan 2, 3-dioxygenase (Tdo; $\mathrm{F}_{1,8}=3.99, \mathrm{P}=0.0808$; Additional file 1, Figure S1B) and solute carrier family 39 (metal ion transporter) member 6 (Slc39a6; $\mathrm{F}_{1,8}=1.902$, $\mathrm{P}=0.2052$; Additional file 1, Figure S1C) in the hippocampus compared with WT mice. Thus alpha-CaMKII over- expressing mice displayed similar changes in gene expressions with alpha-CaMKII (+/-) heterozygous knockout mice although these alterations of gene expression in alpha-CaMKII overexpression mice were not dramatic compared with those in alpha-CaMKII (+/-) heterozygous knockout mice (See 17). Further studies are required to understand the relationships between changes in these gene expressions and hippocampal function in mutant mice showing down or up regulation of alpha CaMKII level in hippocampus.

\section{Increased anxiety-like behavior in alpha-CaMKII overexpressing mice}

Previous studies have demonstrated that alpha-CaMKII $(+/-)$ heterozygous knockout mice display a dramatic 
decrease in anxiety-like behaviors [16,17]. Therefore, to understand the roles of alpha-CaMKII in anxiety-like behaviors, we performed open field, elevated zero maze, light-dark transition and social interaction tests, which enabled us to estimate the anxiety state of alpha-CaMKII overexpressing mice.

We first performed the open field test to measure anxietylike behavior and locomotor activity. Since mice fear novel and open spaces, and generally avoid the center of open fields, this test is used to measure anxiety. alphaCaMKII overexpressing mice exhibited a significantly reduced total path in the open field compared to WT littermates $\left(\mathrm{F}_{1,26}=26.79, \mathrm{P}<0.0001\right.$; Figure $\left.2 \mathrm{~A}\right)$. More importantly, these mutant mice exhibited a significant decrease in the percentage of paths in the center of the field $\left(\mathrm{F}_{1,26}=24.206, \mathrm{P}<0.0001\right.$; Figure $\left.2 \mathrm{~B}\right)$. These results suggest that alpha-CaMKII overexpressing mice display a decrease in locomotor activity in a novel environment and an increase in anxiety-like behavior.
We next performed the elevated zero maze test. In this task, since mice generally avoid high open spaces, the duration of time that they spend in the open spaces and the number of times they enter such spaces are thought to reflect their state of anxiety. Similarly with the results in the open field test, alpha-CaMKII overexpressing mice exhibited significantly decreased number of entries into the open section $\left(\mathrm{F}_{1,37}=15.754, \mathrm{P}=0.0003\right.$; Figure $\left.2 \mathrm{C}\right)$ and time spent in the open section $\left(\mathrm{F}_{1,37}=9.190, \mathrm{P}=\right.$ 0.0044 ; Figure 2D) compared with WT mice. These results are consistent with the previous observations in the open field test (Figure 2A, Figure 2B) and suggest that alphaCaMKII overexpressing mice exhibited a significant increase in anxiety-like behavior. We next performed the light-dark transition test. Since mice generally avoid light spaces, the latency until the mice enter the light box from the dark box, the duration that they spend in the light box and the number of transitions they make from the dark box to the light box are thought to reflect their state of anxiety. alpha-CaMKII overexpressing mice exhibited a

\section{Figure 2}
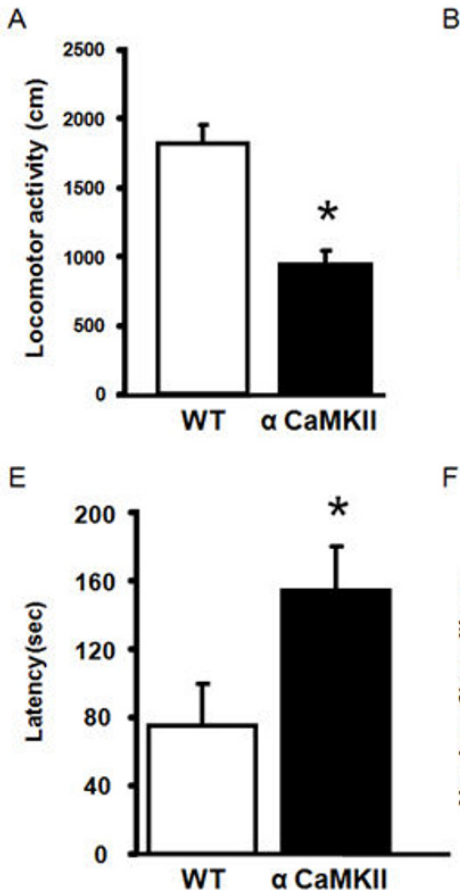

B

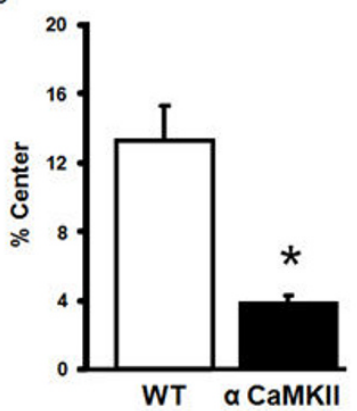

$\mathrm{F}$

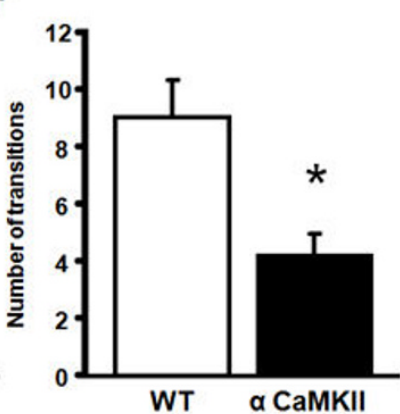

C

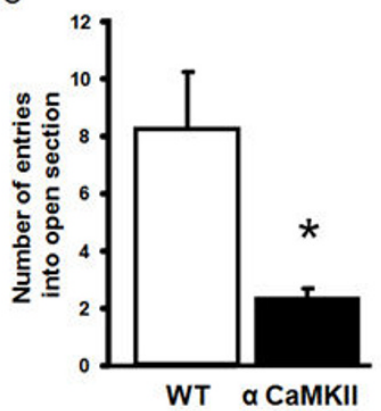

G

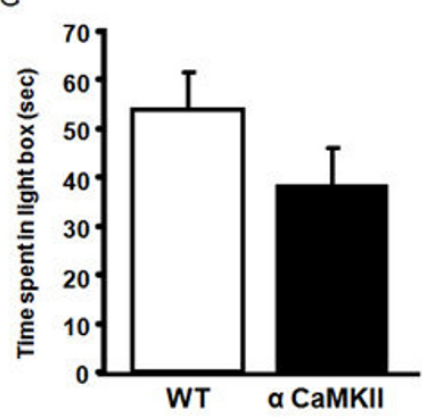

D

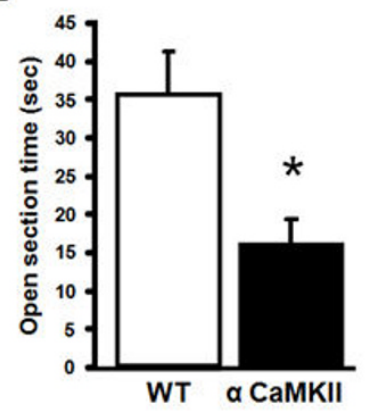

$\mathrm{H}$

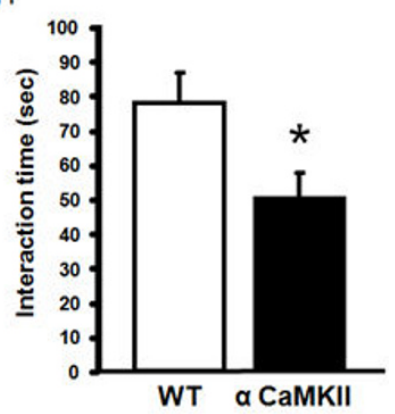

Figure 2

alpha-CaMKII overexpressing mice exhibit an increase in anxiety-like behaviors. (A, B) The total path length (A) and the percent of time spent in the center $(B)$ the open field test, are shown for alpha-CaMKII overexpressing $(n=10)$ and WT mice $(n=10)$. (C, D) The number of entries into the open section $(C)$ and the time spent in the open section (D) in the elevated zero maze test, are shown for alpha-CaMKII $(n=26)$ and WT mice $(n=13)$. $(E-G)$ Latency $(E)$, number of transitions $(F)$ and time spent in light box $(G)$ in light/dark transition test, are shown for alpha-CaMKII $(n=I 3)$ and WT mice $(n=I 3)$. $(H)$ Social interaction time in the social interaction test are shown for alpha-CaMKII $(n=13)$ and WT mice $(n=12)$. The asterisk indicates statistical significance at $\mathrm{P}<0.05$. 
significant increase in the latency until they entered the light box $\left(\mathrm{F}_{1,24}=4.941, \mathrm{P}=0.0359\right.$; Figure $\left.2 \mathrm{E}\right)$ and a significant decrease in the number of transitions they made $\left(\mathrm{F}_{1,24}=9.599, \mathrm{P}=0.049\right.$; Figure $\left.2 \mathrm{~F}\right)$ compared with $\mathrm{WT}$ mice. In addition, we also observed a trend towards to a decrease in the time that alpha-CaMKII overexpressing mice spent in the light box $\left(\mathrm{F}_{1,24}=1.981, \mathrm{P}=0.1721\right.$; Figure $2 \mathrm{G}$ ). Thus, these changes suggest that the up-regulation of alpha-CaMKII expression leads to an increase in anxiety.

An increase in the state of anxiety is thought to lead to a decrease of social behavior such as social investigation [20]. Therefore, we finally measured the social interactions of male alpha-CaMKII overexpressing mice with juvenile male mice. These transgenic mice displayed a significantly shorter social interaction time with juvenile mice compared with WT mice during a $3 \mathrm{~min}$ social interaction test $\left(\mathrm{F}_{1,23}=6.379, \mathrm{P}=0.0189\right.$; Figure $\left.2 \mathrm{H}\right)$. This result indicates that alpha-CaMKII overexpressing mice display a decrease in social behavior. This decrease in social behavior might reflect the increase in anxietyrelated behavior.

Collectively, all of these observations suggest that up-regulation of alpha-CaMKII expression in the forebrain leads to an increase in anxiety. Interestingly, these phenotypes of anxiety-like behaviors observed in alpha-CaMKII overexpressing mice are the opposite to those observed in alpha-CaMKII (+/-) heterozygous knockout mice that display a dramatic decrease in anxiety-like behaviors $[16,17]$. Therefore, these comparisons between alpha-CaMKII overexpressing and alpha-CaMKII (+/-) heterozygous knockout mice suggest that the expression level of alphaCaMKII positively correlates with the anxiety state; more alpha-CaMKII expression leads to an increase in anxiety.

\section{Decreased locomotor activity of alpha-CaMKII overexpressing mice in their home cages}

A recent study has shown that alpha-CaMKII (+/-) heterozygous knockout mice display increased locomotor activity and periodic mood-change-like behavior in their home cages [17]. Our results indicate that alpha-CaMKII overexpressing mice display a decrease in locomotor activity in a novel environment (Figure 2A). Therefore, to examine whether this decrease in locomotor activity observed in the alpha-CaMKII overexpressing mice is specific for novel environments, we measured the locomotor activity of alpha-CaMKII overexpressing mice in their home cages. In contrast to the results observed for alpha-CaMKII (+/-) heterozygous knockout mice, alpha-CaMKII overexpressing mice exhibited a significant decrease in locomotor activity in their home cages during the day and in both the light and dark phases (day, $\mathrm{F}_{1,22}=17.419, \mathrm{P}=0.0004$; light phase, $\mathrm{F}_{1,22}=15.085 \mathrm{P}=0.0008$; dark phase, $\mathrm{F}_{1,22}=$ $14.071, \mathrm{P}=0.0011$; Figure 3 ). These results suggest that the decrease in locomotor activity observed in alphaCaMKII overexpressing mice in the open field test is not due to the response to the novel environment. These contrasting observations between alpha-CaMKII (+/-) heterozygous knockout and overexpressing mice suggest that the expression level of alpha-CaMKII negatively correlates with locomotor activity.

\section{Motor coordination of alpha-CaMKII overexpressing mice} It is possible that the abnormal emotional behaviors, especially the decrease in locomotor activity in a novel environment and their home cages observed in alphaCaMKII overexpressing mice is due to impaired motor performance. To examine this possibility, we subjected mice to the accelerating rotarod test. Our results showed that alpha-CaMKII overexpressing and WT mice displayed a comparable degree of motor performance (day $1, \mathrm{~F}_{1,27}=$ $0.719, \mathrm{P}=0.4041$; day $2, \mathrm{~F}_{1,27}=0.006 \mathrm{P}=0.9369$; day 3 , $\mathrm{F}_{1,27}=1.45, \mathrm{P}=0.239$; Figure 4), suggesting that up-regulation of alpha-CaMKII expression in the forebrain did not affect motor coordination. Thus, the decrease in locomotor activity observed in alpha-CaMKII overexpressing mice was not likely to have been due to decreased motor performance.

\section{Increased offensive aggression in alpha-CaMKII overexpressing mice}

Previous studies have demonstrated that alpha-CaMKII $(+/-)$ heterozygous knockout mice display increased defensive aggression but normal offensive aggression [16]. Interestingly, alpha-CaMKII overexpressing mice also showed increased aggression towards their cage mates. Therefore, to further understand the role of alphaCaMKII in aggressive behavior, we performed the resident-intruder test. To assess offensive aggression, resident mice were individually housed for 4 weeks prior to the introduction of a WT male intruder mouse that had previously been housed in a group of five animals. alpha-CaMKII overexpressing resident mice showed significantly higher offensive aggression, as assessed by their attack latency $\left(\mathrm{F}_{1,24}=9.939, \mathrm{P}=0.0043\right.$; Figure $\left.5 \mathrm{~A}\right)$ and number of attacks $\left(\mathrm{F}_{1,24}=4.463, \mathrm{P}=0.0452\right.$; Figure $\left.5 \mathrm{~B}\right)$, compared with WT resident mice. To assess defensive aggression, mice that had previously played the role of a resident animal became the intruder; a different group of WT mice that had been individually housed for 4 weeks, were used as the residents. alpha-CaMKII overexpressing intruder mice showed a comparable attack latency $\left(\mathrm{F}_{1,18}=0.01, \mathrm{P}\right.$ $=0.92 ;$ Figure $5 \mathrm{C})$ and number of attacks $\left(\mathrm{F}_{1,18}=0.01, \mathrm{P}\right.$ $=0.9208$; Figure 5D) with WT intruder mice, indicating that alpha-CaMKII overexpressing mice display normal defensive aggression. These results indicate that in contrast to alpha-CaMKII (+/-) heterozygous knockout mice, alpha-CaMKII overexpressing mice display increased offensive aggression but normal defensive aggression. 


\section{Figure 3}

A
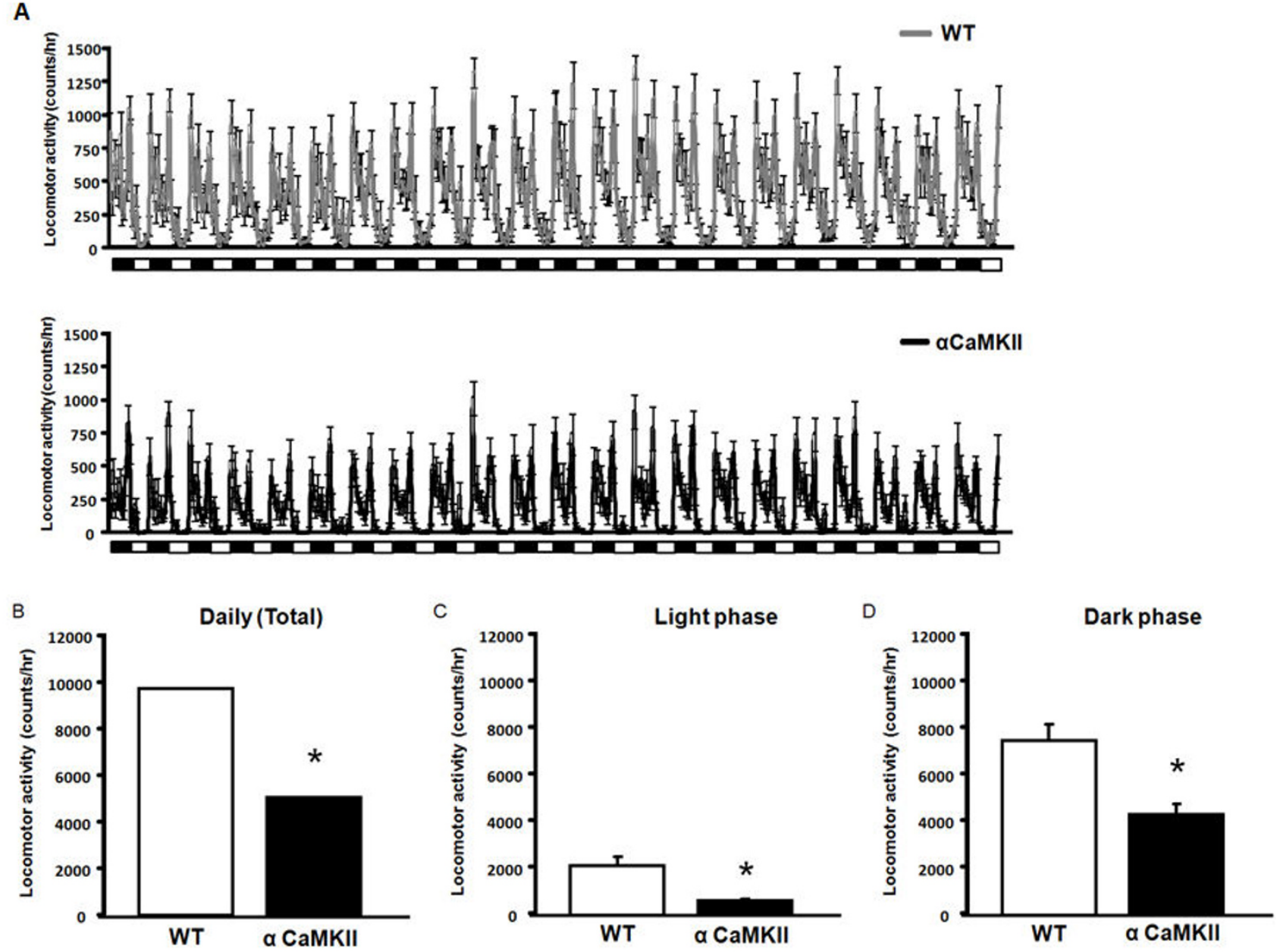

Figure 3

alpha-CaMKII overexpressing mice exhibit a decrease in locomotor activity in their home cages. (A-B) Changes in locomotor activity of WT (A) and alpha-CaMKII overexpressing (B) in their home cages. (C-E) Locomotor activity during a day (C) and light (D) and dark (E) phases are shown for alpha-CaMKII overexpressing $(n=I I)$ and WT mice $(n=I 3)$. The asterisk indicates statistical significance at $\mathrm{P}<0.05$.

\section{Discussion}

Alpha-CaMKII is believed to play essential roles in synaptic plasticity and learning/memory [1-5]. However, previous studies have demonstrated that alpha-CaMKII (+/-) heterozygous knockout mice display a dramatic decrease in fear behavior and anxiety-like behavior, and increased defensive aggression but normal offensive aggression $[16,17]$. These findings indicate that alpha-CaMKII regulates multiple brain functions including not only learning/memory but also emotional behaviors. In this study, to further understand the roles of alpha-CaMKII in emotional behavior, we generated transgenic mice overexpressing alpha-CaMKII in the forebrain and analyzed the behavioral phenotypes of these transgenic mice. In contrast to the behavioral phenotypes observed in alpha-
CaMKII (+/-) heterozygous knockout mice, alpha-CaMKII overexpressing mice exhibited a significant increase in anxiety-like behaviors in the open field, elevated zero maze and light-dark transition tests, suggesting that upregulation of alpha-CaMKII expression leads to an increase in anxiety. Furthermore, these overexpressing mice also display increased offensive aggression but normal defensive aggression. Therefore, taken together with previous observations $[16,17]$, our findings indicate that the expression level of alpha-CaMKII has a strong impact on emotional behavior, especially anxiety-like behavior and aggression (Figure 6).

alpha-CaMKII is believed to be an essential molecule for learning/memory [1-5]. Indeed, alpha-CaMKII (-/-) 


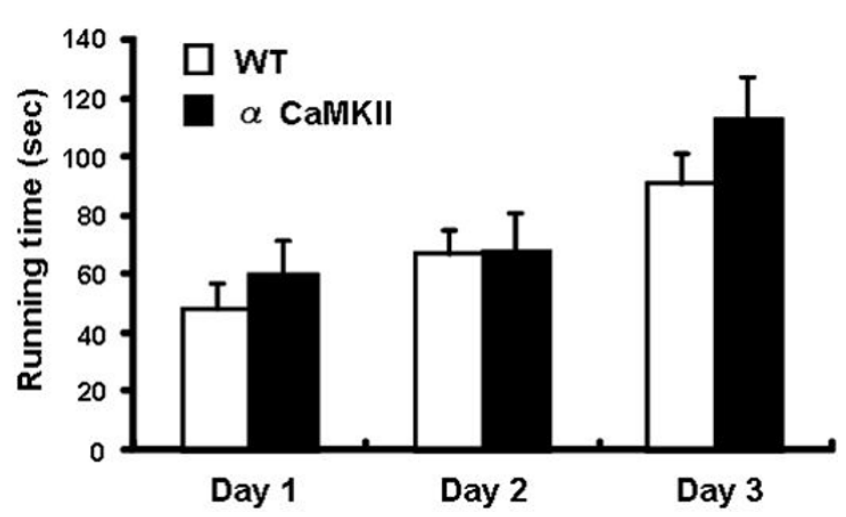

Figure 4

alpha-CaMKII overexpressing mice exhibit normal motor performance in the rotarod test. Running time is shown for alpha-CaMKII overexpressing $(n=13)$ and WT mice $(n=16)$.

homozygous knockout mice showed deficits in learning [8]. Furthermore, mutant mice lacking the autonomous activity of alpha-CaMKII (T286A mutant mice) also exhibited impaired learning [9]. However, up-regulation of alpha-CaMKII expression or activity does not simply result in the expected gain of function-effects such as the enhancement of leaning; transgenic mice overexpressing a constitutively active mutation of alpha-CaMKII (T286D) or wild-type alpha-CaMKII in the forebrain exhibited impaired learning and memory formation [11-15]. Consistent with previous studies [14], we also observed that the alpha-CaMKII overexpressing mice analyzed in this study displayed deficits in memory formation (data not shown). These observations are in contrast to the case of CaMKIV which positively regulates memory formation; a null mutation of the CaMKIV gene impairs memory consolidation [21-23], while forebrain-specific overexpression of this kinase enhances memory consolidation $[19,24]$. These previous studies suggest that alpha-CaMKII levels do not simply correlate with learning/memory performance $[14,15]$. However, we cannot exclude other possibilities; a moderate up-regulation of alpha-CaMKII expression levels or activity is required for the enhancement of learning/memory. Additionally, transgenes used in our and other studies lack 3'-untranslated region of alpha-CaMKII targeting signals for the localization of alpha-CaMKII mRNA to the dendrites [11-15,25]. Therefore, the possibility still remains that this lack of 3'UTR of alpha-CaMKII in transgenes resulted in abnormal intracellular localization of overexpressed alpha-CaMKII in cell bodies, thereby leading to deficits in learning/memory and abnormal emotional behaviors. In contrast, alphaCaMKII overexpressing mice and alpha-CaMKII (+/-) heterozygous knockout mice displayed opposite emotional behavioral phenotypes; alpha-CaMKII overexpressing mice displayed an increase in anxiety-like behavior and a
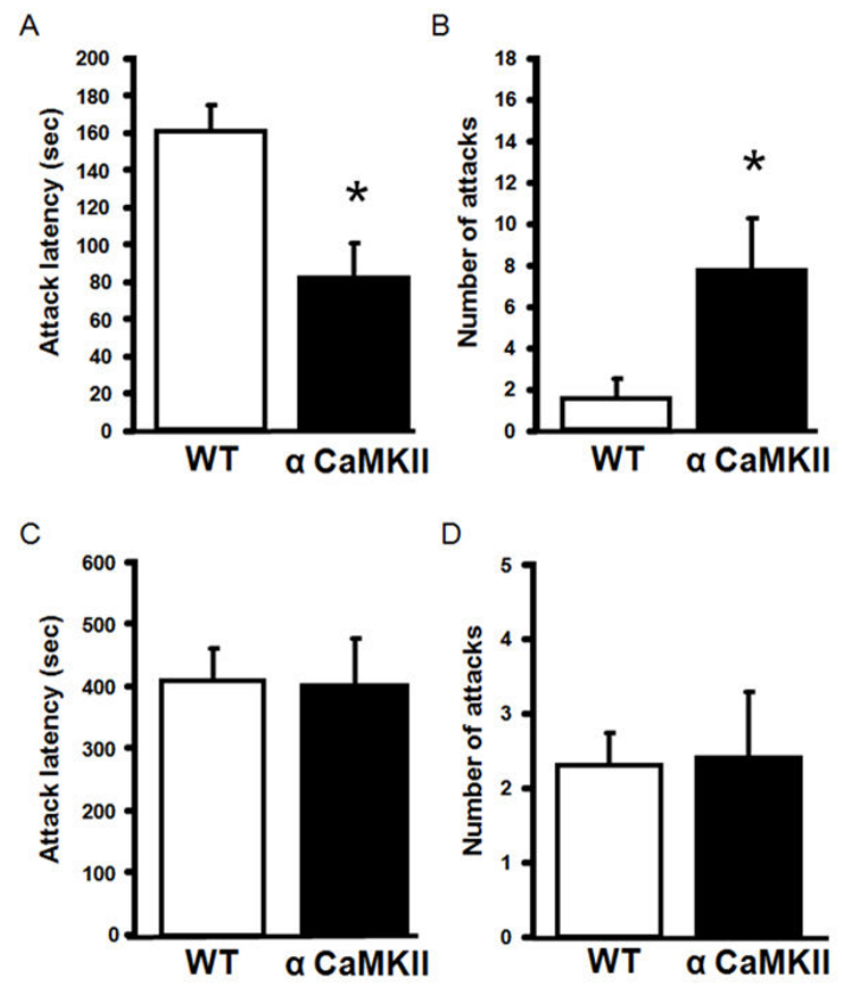

Figure 5

alpha-CaMKII overexpressing mice display an increase in offensive aggression in the residentintruder test. (A, B) offensive aggression. (C, D) defensive aggression. The latency to first attack bite (attack latency; $A$, $C)$ and the number of attacks $(B, D)$ are shown for alphaCaMKII overexpressing $(n=10)$ and WT mice $(n=10)$. The asterisk indicates statistical significance at $\mathrm{P}<0.05$.

decrease in locomotor activity, while alpha-CaMKII (+/-) heterozygous knockout mice display a decrease in anxietylike behavior and an increase in locomotor activity $[16,17]$. Therefore, these comparisons strongly suggest that the expression level of alpha-CaMKII might determine the emotional state, and especially that the expression level of alpha-CaMKII positively correlates with the state of anxiety-like behavior (Figure 6).

Previous studies also demonstrated that alpha-CaMKII (+/ -) heterozygous knockout mice displayed aggressive behaviors towards to their cage mates $[16,17]$. Further investigation demonstrated that these mice displayed an increase in defensive aggression but displayed normal offensive aggression [16], which seems to reflect a decrease in the fear response observed in these mice [16]. Interestingly, we also observed that alpha-CaMKII overexpressing mice exhibit a high frequency of fighting in their home cages, suggesting that both up-regulation and down-regulation of alpha-CaMKII leads to an increase in aggressive behavior. To answer why both groups of mice 
A
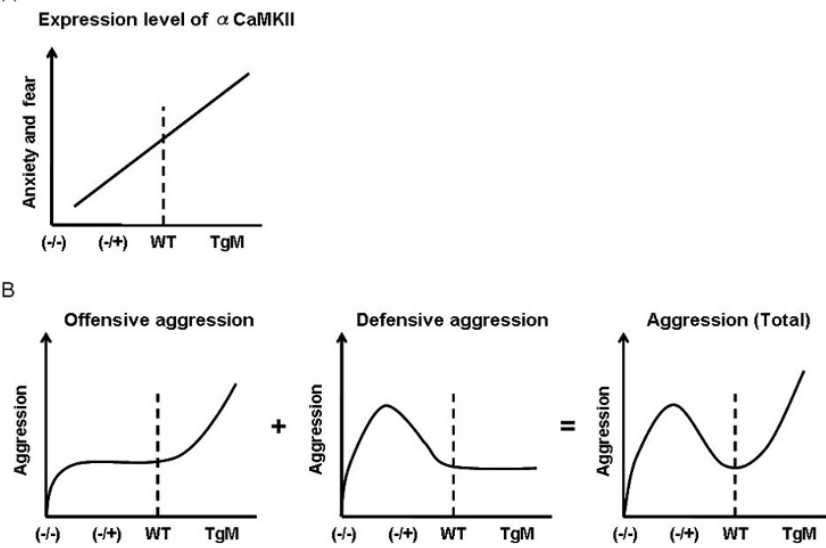

Figure 6

The relationship between the expression level of alpha-CaMKII in the forebrain and anxiety, fear and aggression. (A) Expression level of alpha-CaMKII positively correlates with anxiety-like and fearful behaviors. (B) alphaCaMKII (+/-) heterozygous knockout and alpha-CaMKII overexpressing mice display an increase in defensive and offensive aggression, respectively.

display similarities of aggressive behavior, we analyzed the aggressive behavior of the alpha-CaMKII overexpressing mice using the resident-intruder test. In contrast to the observations in alpha-CaMKII (+/-) heterozygous knockout mice, alpha-CaMKII overexpressing mice exhibited an increase in offensive aggression but displayed normal defensive aggression. This increase in offensive aggression is thought to reflect the increase in anxiety observed in alpha-CaMKII overexpressing mice (Figure 2). Therefore, the comparison of phenotypes between alpha-CaMKII $(+/$ -) heterozygous knockout and overexpression mice indicates that both an increase and decrease in expression levels of alpha-CaMKII leads to an increase in aggressive behavior, but the aggression state is different between upregulation and down-regulation of alpha-CaMKII expression levels in the forebrain; up-regulation and down-regulation of alpha-CaMKII levels increases offensive and defensive aggression, respectively (Figure 6). These changes in the aggression state seem to reflect opposite anxiety states between the mice up-regulating and downregulating the expression levels of alpha-CaMKII.

Previous studies have pointed out that alpha-CaMKII (+/) heterozygous knockout mice display behavioral abnormalities similar to the symptoms observed in patients with psychiatric diseases such as schizophrenia and bipolar disorders [17]. In this study, we observed that alphaCaMKII overexpressing mice displayed an increase in anxiety-like behaviors. Comparison between this and previous studies suggest that changes in alpha-CaMKII expression levels strongly affects the emotional state; up- regulation and down-regulation of CaMKII causes a increase or decrease, respectively, in anxiety-like behavior. Therefore, it is possible that the abnormal alteration of alpha-CaMKII expression levels is implicated in psychiatric disorders. It is important to investigate the molecular mechanisms responsible for the regulation of alpha-CaMKII expression in vivo and identify the factors that affect the expression levels of alpha-CaMKII.

\section{Conclusion}

In summary, we generated transgenic mice overexpressing alpha-CaMKII in the forebrain and showed that these transgenic mice displayed an increase in anxiety-like behaviors and offensive aggression; both of which are in contrast to the emotional behavior phenotypes observed in alpha-CaMKII (+/-) heterozygous knockout mice. From these findings, we suggest that the expression levels of alpha-CaMKII positively correlate with the anxiety state and strongly affect aggression state. Therefore, changes in the expression levels of alpha-CaMKII might be associated with psychiatric disorders such as anxiety, depression and bipolar disorders.

\section{Methods \\ Animals}

Mice were housed in cages of five or six, maintained on a $12 \mathrm{~h} \mathrm{light/dark} \mathrm{schedule} \mathrm{and} \mathrm{allowed} \mathrm{ad} \mathrm{libitum} \mathrm{access} \mathrm{to}$ food and water in their home cages. All experiments were conducted during the light phase of the cycle in an illuminated testing room, according to the Guide for the Care and Use of Laboratory Animals, Japan Neuroscience Society, and Tokyo University of Agriculture. Mice were at least 8 weeks of age when tested. All experiments were conducted blind to the treatment condition of the mouse. Animal behavior was recorded using a video camera.

\section{Generation of transgenic mice}

To introduce MluI restriction sites at the $5^{\prime}$ and $3^{\prime}$ ends of the cDNA encoding alpha-CaMKII, a plasmid containing wild-type CaMKII (kindly provided by Dr. Alcino J. Silva) was amplified by PCR with the following primers: forward primer,

GGGGATATCGCCGCCACCATGGACTACAAGGACGAC GATGACAAACTCAAAACGCGTGCTACCATCACCT-

GCACCCGATTC, and reverse primer, GGGGATATCACGCGTTCAATGCGGCAGGACGGA. The resulting fragment was ligated with a $12.5 \mathrm{~kb} \mathrm{Mlu}$ I fragment containing the alpha-CaMKII promoter, a hybrid intron in the $5^{\prime}$ untranslated leader sequence, Kozak consensus sequence, FLAG-tag sequence following an initiation codon and the SV40 polyadenylation signals from pMM403-CaMKIV $[18,19]$, generating a new construct, pMM403-alpha-CaMKII. The transgene contained an alphaCaMKII promoter, a hybrid intron in the 5 ' untranslated leader sequence, the coding region of alpha-CaMKII and a 
SV40 polyadenylation [poly (A)] signal. pMM-403-alphaCaMKII was digested with $S f i$ and transgenic mice were generated by injecting the purified insert into the pronuclei of C57BL/6N mice (Charles River, Kanagawa, Japan). Genotyping was performed by Southern blot analysis using a probe derived from a $1.1 \mathrm{~kb}$ fragment produced by EcoRV/NotI digestion [SV40 poly (A) probe] from pNN265-LBDG521R-CREBS133A [18].

\section{In situ hybridization analysis}

In situ hybridization was preformed as previously described $[26,27]$. Brains were frozen on dry ice and sectioned on a cryostat at $16 \mu \mathrm{m}$. The sections were fixed in $4 \%$ paraformaldehyde in phosphate-buffered saline (PBS) and washed in PBS containing $0.1 \%$ diethylpyrocarbonate. After two washes in $5 \times$ saline sodium citrate (SSC), the sections were incubated in prehybridization solution [ $50 \%$ formamide, $5 \times$ SSC, $2 \%$ DNA-blocking solution (Roche Diagnostics, Mannheim, Germany) and $40 \mu \mathrm{g} / \mathrm{ml}$ salmon testis DNA] for $2 \mathrm{~h}$ at $58^{\circ} \mathrm{C}$ and hybridized in the above prehybridization solution containing digoxigenin (DIG)-labelled anti-sense RNA probes overnight at $58^{\circ} \mathrm{C}$. After washes in $2 \times$ SSC and $0.1 \times$ SSC at $65^{\circ} \mathrm{C}$, the sections were rinsed in antibody buffer $(100$ $\mathrm{mM}$ Tris $\mathrm{pH} 7.5,150 \mathrm{mM} \mathrm{NaCl}$ and $0.5 \%$ DNA-blocking solution) and incubated in antibody buffer containing a 1:5000 dilution of anti-DIG antibody coupled with alkaline phosphatase (AP) (Roche Diagnostics) for $2 \mathrm{~h}$ at room temperature. After two washes in antibody solution and a wash in Tris-buffered saline (TBS) buffer containing $100 \mathrm{mM}$ Tris $\mathrm{pH} 9.5,100 \mathrm{mM} \mathrm{NaCl}$ and $50 \mathrm{mM} \mathrm{MgCl}{ }_{2}$, the sections were stained in TBS buffer containing nitroblue tetrazolium and 5-bromo-4-chloro-3-indoryl phosphate (Roche Diagnostics) overnight at room temperature. The sections were rinsed in water and 95\% ethanol and mounted. Control experiments were performed using sense DIG-labeled probes. Sense and antisense probes were generated from the $1.1 \mathrm{~kb}$ EcoRV-NotI fragment of pNN-LBDG521R-CREBS133A cloned into pBluescriptII-SK (Stratagene, CA) using an in vitro transcription kit (Ambion, TX).

\section{Quantitative real-time polymerase chain reaction (qRT- PCR)}

qRT-PCR was performed in an ABI PRISM 7000 with SYBR green PCR master mix (Applied Biosystems, CA) according to the manufacturer's protocol. Amplification of the single PCR product was confirmed by monitoring the dissociation curve and electrophoresis on $1.2 \%$ agarose gels stained with ethidium bromide. The reaction was first incubated at $50^{\circ} \mathrm{C}$ for $2 \mathrm{~min}$, then at $95^{\circ} \mathrm{C}$ for $10 \mathrm{~min}$, followed by 40 cycles of $95^{\circ} \mathrm{C}$ for $15 \mathrm{sec}$ and $60^{\circ} \mathrm{C}$ for $1 \mathrm{~min}$. All measurements were performed in triplicate. Levels of GAPDH mRNA was used to normalize the relative expression levels of target mRNA. The PCR primers used were as follows ( $5^{\prime}$ to $\left.3^{\prime}\right)$ : Tdo2 forward, CTATGAGTGGGT-
GCCCGTTT; Tdo2 reverse, CCTCCTTTGCTGGCTCTGTT; Dsp forward, GCCGTCAAAATCACCAACC; Dsp reverse, CCATCCAGCACATCCCTCT; Npnt forward, CGCTATGGAGGCAGGATTG; Npnt reverse, TCCGTGTTTGCACTGTGGTT; Pnck forward, ATGGTCTCTGACTTTGGCCTGT; Pnck reverse, TTCCCGTAGGGTTTCTGCTC; Slc39a6 forward, CAGCAAGTGAGAAGAAGGCAGA; Slc39a6 reverse, ACCAAGATGACTCCCAGCAGA; GAPDH forward, ATGGCCTTCCGTGTTCCTAC; GAPDH reverse, GCCTGCTTCACCACCTTCTT.

\section{Western blot analysis}

Samples of the hippocampus and cortex of alpha-CaMKII and WT littermate control mice were isolated, homogenized in SDS buffer (0.1 M dithiothreitol (DTT), 2\% SDS

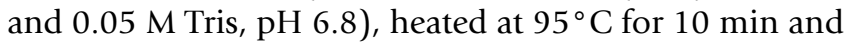
centrifuged at $4^{\circ} \mathrm{C}$ for $10 \mathrm{~min}$. The supernatants $(50 \mu \mathrm{g}$ of total protein) were analyzed by western blotting. Western blotting, using an anti-alpha-CaMKII antibody (1:2000; Santa Cruz Biotechnology, CA), anti-phospho-alphaCaMKII T286 antibody (1:2000 Promega, WI), and an anti-alpha-tubulin antibody (1:1000; Santa Cruz Biotechnology) were performed as previously described [28]. Positive antibody binding was visualized using ECL chemiluminescence (GE Healthcare Bio-Sciences Corp., Piscataway, NJ), and membranes were analyzed with the Lumi-imager TM chemiluminescence detection system (Roche Diagnostics, Basel, Switzerland). The amount of protein in lanes was confirmed to be comparable by reprobing with an anti-alpha-tubulin antibody.

\section{Behavioral procedures}

Open field test [29]

Mice were placed into the center of a square open field chamber $(40 \mathrm{~cm}$ long $\times 40 \mathrm{~cm}$ wide $\times 40 \mathrm{~cm}$ high $)$ that was surrounded by white acrylic walls. The total length of the path the mouse traveled (locomotor activity) and the time it spent in a center square $(24 \mathrm{~cm} \times 24 \mathrm{~cm}$; \% center $)$ were measured over the course of 5 min using an automatic monitoring system (TARGET 2, Neuroscience Inc., Tokyo, Japan).

\section{Elevated zero maze test [29]}

The zero maze consisted of a circular path $(5.5 \mathrm{~cm}$ width, inner diameter of $46 \mathrm{~cm}$ ) that had two open and two closed sections (wall was $15 \mathrm{~cm}$ high) and was elevated 50 $\mathrm{cm}$ above the floor. Mice were initially placed in the closed section and their behavior was observed for $5 \mathrm{~min}$. The length of time that they spent in the open section (open section time) and the number of times they entered into the open section with two or four paws (number of entries into open section) were measured.

\section{Light/dark transition test}

The apparatus used for the light/dark transition test consisted of a box $(21 \times 42 \times 25 \mathrm{~cm})$ divided into two sections 
of equal size by a partition with a door (O'Hara \& Co., Ltd., Tokyo, Japan). One box is brightly illuminated by white diodes (390 lux), whereas the other box is dark (2 lux). Mice were placed into the dark side and the door was opened $3 \mathrm{sec}$ after the mouse was detected by the infrared camera. The door was used so that the mice do not enter the light box immediately after release with their motivation to escape from the experimenter, since the latency to enter the light box may serve as an index of anxiety-like behavior. Mice were allowed to move freely between the two boxes for $5 \mathrm{~min}$. The distance traveled in each box, the number of transitions, the time spent in each box and the latency to enter the light box were measured by Image LD4 (O'Hara \& Co., Ltd.), modified software based on the public domain NIH Image program (developed at the U.S. National Institutes of Health and available on the Internet at http://rsb.info.nih.gov/nih-image/).

\section{Social interaction test [19]}

Each male adult subject (alpha-CaMKII overexpressing mouse or WT littermate) was placed in a measuring cage and allowed to stay for $60 \mathrm{~min}$. A male juvenile was introduced into the cage and the amount of time spent in social interaction (e.g. grooming, licking, sniffing, and crawling over or under) with the tested animal was recorded during a 3 min session.

\section{Locomotor activity monitoring in the home cage}

Daily locomotor activity in the home cage was detected by 124 infrared photocell beams in an adjustable frame placed over the cage for every individual mouse (Biotex, Kyoto, Japan). The animals were maintained on a $12 \mathrm{hr}$ light: 12 hr dark (LD) lighting cycle after habituation for a week before recording.

\section{Rotarod test [29]}

Mice were placed on a rotating drum $(3 \mathrm{~cm}$ in diameter; O'Hara \& Co., Ltd.). The drum was initially rotated at a speed of $4 \mathrm{rpm}$ after which it was accelerated gradually to $40 \mathrm{rpm}$ over the course of $5 \mathrm{~min}$. The amount of time that the mouse remained on the accelerating rod (running time) was recorded as an indicator of their motor performance, on 3 successive days.

\section{Resident-intruder test [29]}

To assess offensive aggression, resident mice were individually housed for 4 weeks prior to the introduction of a WT male intruder mouse, which was previously housed in a group of five animals. When we assess offensive aggression, the resident mouse was paired with the same intruder mouse for $3 \mathrm{~min}$. To assess defensive aggression, mice that had previously played the role of the resident animal became the intruder; a different group of WT mice that had been individually housed for 4 weeks, were used as the residents. When we assess defensive aggression, res- ident mouse was paired with the same intruder mouse for $10 \mathrm{~min}$. The latency to first attack bite (attack latency) and the total number of attacks by the test mice (number of attacks) were measured.

\section{Statistics}

All values are expressed as the mean + SE. Data were analyzed using an analysis of variance (ANOVA) and a singlefactor ANOVA and Newman-Keuls post hoc comparison were used to analyze differences between groups for the behavioral tests.

\section{Competing interests}

The authors declare that they have no competing interests.

\section{Authors' contributions}

SK is responsible for the hypothesis development and overall design of the research and experiment, and supervised the experimental analyses. SK and SHasegawa cowrote the manuscript. SHasegawa, TF, TY, KE, MS, TM and RS performed behavioral analyses. TY and RM generated transgenic mice overexpressing alpha-CaMKII. AK constructed a transgene. SHasegawa, TF and KK performed biochemical analyses. SHomma, SM and KY supervised experimental analyses.

\section{Additional material}

\section{Additional file 1}

Figure S1. Gene expression analyses in hippocampus of alpha-CaMKII overexpressing mice. (A-E) The mRNA expression levels of nephronectin (A), tryptophan 2,3-dioxygenase (B), solute carrier family 39 (metal ion transporter), member $6(C)$, desmoplakin $(D)$ and pregnancy upregulated non-ubiquitously expressed CaM kinase (E) in the hippocampus of alpha-CaMKII overexpressing $(n=5)$ and WT mice $(n=5)$. The asterisk indicates statistical significance at $P<0.05$.

Click here for file

[http://www.biomedcentral.com/content/supplementary/17566606-2-6-S1.pdf]

\section{Acknowledgements}

S.K. was supported by Grant-in-Aids for Scientific Researches (I8580I29 and 20380078), High Technology Research and Priority Areas-Molecular Brain Science- ( 18022038 and 22022039) from the ministry of Education, Culture, Sports, Science and Technology, Japan, Core Research for Evolutional Science and Technology, Japan and the Research Grant for Nervous and Mental Disorders from the Ministry of Health, Labour and Welfare, Japan.

\section{References}

I. Colbran RJ: Protein phosphatases and calcium/calmodulindependent protein kinase II-dependent synaptic plasticity. J Neurosci 2004, 24:8404-8409.

2. Colbran RJ, Brown AM: Calcium/calmodulin-dependent protein kinase II and synaptic plasticity. Curr Opin Neurobiol 2004, 14:318-327. 
3. Lisman J, Schulman H, Cline H: The molecular basis of CaMKII function in synaptic and behavioural memory. Nat Rev Neurosci 2002, 3: $175-190$.

4. Soderling TR: CaM-kinases: modulators of synaptic plasticity. Curr Opin Neurobiol 2000, 10:375-380.

5. Wayman GA, Lee YS, Tokumitsu H, Silva A, Soderling TR: Calmodulin-kinases: modulators of neuronal development and plasticity. Neuron 2008, 59:914-931.

6. Malenka RC, Nicoll RA: Long-term potentiation - a decade of progress? Science 1999, 285: 1870-1874

7. Silva AJ, Stevens CF, Tonegawa S, Wang Y: Deficient hippocampal long-term potentiation in alpha-calcium-calmodulin kinase II mutant mice. Science 1992, 257:201-206.

8. Silva AJ, Paylor R, Wehner JM, Tonegawa S: Impaired spatial learning in alpha-calcium-calmodulin kinase II mutant mice. Science 1992, 257:206-2II.

9. Giese KP, Fedorov NB, Filipkowski RK, Silva AJ: Autophosphorylation at Thr286 of the alpha calcium-calmodulin kinase II in LTP and learning. Science 1998, 279:870-873.

10. Elgersma $Y$, Fedorov NB, Ikonen S, Choi ES, Elgersma M, Carvalho OM, Giese KP, Silva AJ: Inhibitory autophosphorylation of CaMKII controls PSD association, plasticity, and learning. Neuron 2002, 36:493-505

II. Mayford M, Wang J, Kandel ER, O'Dell TJ: CaMKII regulates the frequency-response function of hippocampal synapses for the production of both LTD and LTP. Cell 1995, 81:891-904

12. Bach ME, Hawkins RD, Osman M, Kandel ER, Mayford M: Impairment of spatial but not contextual memory in CaMKII mutant mice with a selective loss of hippocampal LTP in the range of the theta frequency. Cell 1995, 81:905-915.

13. Mayford M, Bach ME, Huang YY, Wang L, Hawkins RD, Kandel ER: Control of memory formation through regulated expression of a CaMKII transgene. Science 1996, 274:1678-1683.

14. Wang H, Shimizu E, Tang YP, Cho M, Kyin M, Zuo W, Robinson DA, Alaimo PJ, Zhang C, Morimoto H, Zhuo M, Feng R, Shokat KM, Tsien JZ: Inducible protein knockout reveals temporal requirement of CaMKII reactivation for memory consolidation in the brain. Proc Natl Acad Sci USA 2003, 100:4287-4292.

15. Cao X, Wang H, Mei B, An S, Yin L, Wang LP, Tsien JZ: Inducible and selective erasure of memories in the mouse brain via chemical-genetic manipulation. Neuron 2008, 60:353-366.

16. Chen C, Rainnie DG, Greene RW, Tonegawa S: Abnormal fear response and aggressive behavior in mutant mice deficient for alpha-calcium-calmodulin kinase II. Science 1994, 266:291-294.

17. Yamasaki N, Maekawa M, Kobayashi K, Kajii Y, Maeda J, Soma M, Takao K, Tanda K, Ohira K, Toyama K, Kanzaki K, Fukunaga K, Sudo Y, Ichinose H, Ikeda M, Iwata N, Ozaki N, Suzuki H, Higuchi M, Suhara T, Yuasa S, Miyakawa T: Alpha-CaMKII deficiency causes immature dentate gyrus, a novel candidate endophenotype of psychiatric disorders. Mol Brain 2008, I:6.

18. Kida S, Josselyn SA, de Ortiz SP, Kogan JH, Chevere I, Masushige S, Silva AJ: CREB required for the stability of new and reactivated fear memories. Nat Neurosci 2002, 5:348-355.

19. Fukushima $H$, Maeda R, Suzuki R, Suzuki A, Nomoto M, Toyoda $H$, Wu LI, Xu H, Zhao MG, Ueda K, Kitamoto A, Mamiya N, Yoshida T, Homma S, Masushige S, Zhuo M, Kida S: Upregulation of calcium/ calmodulin-dependent protein kinase IV improves memory formation and rescues memory loss with aging. I Neurosci 2008, 28:9910-9919.

20. File SE, Seth P: $\mathbf{A}$ review of $\mathbf{2 5}$ years of the social interaction test. Eur J Pharmacol 2003, 463:35-53.

21. Kang H, Sun LD, Atkins CM, Soderling TR, Wilson MA, Tonegawa S: An important role of neural activity-dependent CaMKIV signaling in the consolidation of long-term memory. Cell 200I, 106:77I-783.

22. Ho N, Liauw JA, Blaeser F, Wei F, Hanissian S, Muglia LM, Wozniak DF, Nardi A, Arvin KL, Holtzman DM, Linden DJ, Zhuo M, Muglia LJ, Chatila TA: Impaired synaptic plasticity and cAMP response element-binding protein activation in $\mathrm{Ca} 2+/$ calmodulindependent protein kinase type IV/Gr-deficient mice. J Neurosci 2000, 20:6459-6472.

23. Wei F, Qiu CS, Liauw J, Robinson DA, Ho N, Chatila T, Zhuo M: Calcium calmodulin-dependent protein kinase IV is required for fear memory. Nat Neurosci 2002, 5:573-579.
24. Wu LJ, Zhang $X H$, Fukushima $H$, Zhang F, Wang $\mathrm{H}$, Toyoda $\mathrm{H}$, Li BM, Kida S, Zhuo M: Genetic enhancement of trace fear memory and cingulate potentiation in mice overexpressing $\mathrm{Ca2}+/ \mathrm{cal}-$ modulin-dependent protein kinase IV. Eur J Neurosci 2008, 27:1923-1932.

25. Mayford M, Baranes D, Podsypanina K, Kandel ER: The 3'-untranslated region of CaMKII alpha is a cis-acting signal for the localization and translation of mRNA in dendrites. Proc Natl Acad Sci USA 1996, 93: I3250-I 3255.

26. Jalabi W, Cerghet M, Skoff RP, Ghandour MS: Detection of oligodendrocytes in tissue sections using PCR synthesis of digoxigenin-labeled probes. J Histochem Cytochem 2003, 51:913-919.

27. Uchida S, Sakai S, Furuichi T, Hosoda H, Toyota K, Ishii T, Kitamoto A, Sekine M, Koike K, Masushige S, Murphy G, Silva AJ, Kida S: Tight regulation of transgene expression by tetracycline-dependent activator and repressor in brain. Genes Brain Behav 2006, 5:96-106.

28. Hosoda H, Motohashi J, Kato H, Masushige S, Kida S: A BMALI mutant with arginine 91 substituted with alanine acts as a dominant negative inhibitor. Gene 2004, 338:235-24I.

29. Uchida S, Kitamoto A, Umeeda H, Nakagawa N, Masushige S, Kida S: Chronic reduction in dietary tryptophan leads to changes in the emotional response to stress in mice. I Nutr Sci Vitaminol (Tokyo) 2005, 5I: 175-I8I.
Publish with Bio Med Central and every scientist can read your work free of charge

"BioMed Central will be the most significant development for disseminating the results of biomedical research in our lifetime."

Sir Paul Nurse, Cancer Research UK

Your research papers will be:

- available free of charge to the entire biomedical community

- peer reviewed and published immediately upon acceptance

- cited in PubMed and archived on PubMed Central

- yours - you keep the copyright

Submit your manuscript here:

http://www.biomedcentral.com/info/publishing_adv.asp
BioMedcentral 